\title{
Gradient Optimisation Methods for the Positioning of Active Noise Control Actuators in Enclosures
}

\author{
G.N. Charalampopoulos, S.T. Mouzakitis and C.G. Provatidis \\ National Technical University of Athens (NTUA), School of Mechanical Engineering, \\ Mechanical Design and Control Systems Section, 9 Iroon Polytechniou st., 157 73, Athens, Greece
}

(Received 5 April 2004; accepted 16 July 2004)

\begin{abstract}
This paper discusses the problem of finding the optimum locations and strengths of a number of secondary sources in an interior active noise control problem. The analysis of the acoustic field is performed using the finite element method, while the positions and strengths of the secondary sources are determined by various optimisation schemes. These schemes are compared to each other in terms of computational effort and efficient prediction of the global optimum when tested in two-dimensional rectangular cavities. The results indicate that a new optimisation method, based on the combination of evolution and deterministic Newton methodology, is best used for this type of problem in most cases.
\end{abstract}

\section{INTRODUCTION}

The achievement of acceptably low noise conditions in a room, which is often called noise control of acoustic enclosures, can be principally achieved in two ways: first, by using passive means, in which case, very satisfactory results can be achieved by using sound-absorbing materials, however, the cost becomes high, and the performance is significantly degraded, when the acoustic wavelengths of the noise source are comparable in size to the dimension of the enclosure, especially below the Schroeder frequency. Second, noise control can be achieved by active noise control (ANC), which is currently well known as the most effective technique in the relatively low frequency range. Physically, ANC is the cancellation of a sound wave by adding a phase inversed sound wave.

Concerning ANC, many applications have been tested and analysed in several diverse problems ${ }^{1}$ in order to reduce sound levels within a confined space. Methods of active noise attenuation primarily fall into two categories: reduction of the noise level in a specified region or direction ${ }^{2}$ and reduction of total noise power ${ }^{3}$ in exterior or enclosed sound fields. Recent advances in technology provoked a catholic change in ANC, and excellent survey papers are reviewed. Wright and Vuksanovic ${ }^{\mathbf{4}, 5}$ developed a controlled acoustic shadow generated by a wall of secondary sources to reduce unwanted sound in the direction of an unconfined complaint area. It was demonstrated that the power output could be reduced to zero if the secondary source is very close to the primary source. In some other papers, Bullmore, Nelson and Elliot $^{6-8}$ attempted to predict the best theoretical positions of the secondary sources based on how efficiently the total time-averaged acoustic potential energy in an enclosed noise field was reduced by these sources, by means of controlling the analytically calculated acoustic modes of the field.

The above referenced engineering problem has also been formulated mathematically as an optimisation problem, and some efforts have been made to face it, with limited success. Specific arrangements of dimensions and geometry forms have been tested for positioning the secondary sound source as well as its strength or/and working frequency range. Some researchers have developed optimisation algorithms to face the peculiarities of this specific problem based on the experience of previous trials with classic optimisation methods. ${ }^{9-14}$ Their effort is mainly divided in two domains: the selection of the secondary source locations among a set of predefined configuration options (which is basically a discrete optimisation problem and is solved primarily by genetic algorithms), and the calculation of the secondary source locations by solving a continuous optimisation problem (in which the possible secondary sources locations are not predefined but constitute the continuous optimisation variables). More recent research by control theorists has additionally incorporated sensors and feedback ideas into similar methodologies, using simulation models to grade the performance of complete actual ANC systems. ${ }^{15}$ Although these papers present realistic simulations of actual ANC systems (and despite the simplicity of the cases examined), it is not always desirable to examine both the physical and the algorithmic design of an ANC system at the same time, especially when this costs heavily on the computational time required by an optimisation procedure.

This paper continues previous research ${ }^{\mathbf{1 6}, 17}$ by further investigating the performance of widely accepted mathematical optimisation techniques, incorporated in MATLAB, for ANC problems of secondary source placement. Moreover, a new global optimisation methodology, based on the combination of evolution and deterministic Newton methodology, is successfully applied to rectangular acoustic enclosures.

\section{MODELLING TECHNIQUES}

The problem of optimising the secondary source locations in ANC problems requires the direct or indirect calculation of the pressure in the treated enclosure. A widely used (indirect) methodology is the one based on the modal analysis of the enclosure, either analytically, ${ }^{6,7}$ or using numerical algorithms,${ }^{14,17}$ which relates the optimum amplitude of the secondary sources to their locations. However, in order to calculate the optimum amplitude of the sources, as stated by Bai 